\title{
Der Isotopieeffekt bei der elektrolytischen Wanderung der Kalium-Ionen in geschmolzenem Kaliumnitrat
}

\author{
Von Arnold Lundén, Carl Reuterswärd und Nils Sjöberg \\ Aus dem Institut für Physik, Chalmers Technische Hochschule, Göteborg \\ und dem Institut für Physik, Uppsala Universitet, Uppsala \\ (Z. Naturforschg. 10a, 279-281 [1955]: eingegangen am 23. Januar 1955)
}

\begin{abstract}
Durch Elektrolyse von geschmolzenem Kaliumnitrat wurde an der Anode ${ }^{41} \mathrm{~K}$ im Verhältnis zu ${ }^{39} \mathrm{~K}$ angereichert. Die relative Differenz der elektrolytischen Wanderungsgeschwindigkeiten dieser beiden Isotope wurde zu 0,0018 bestimmt. Der Masseneffekt ist $\mu=-0,036$.
\end{abstract}

$\mathrm{D}$ urch Untersuchungen des Isotopieeffekts bei der elektrolytischen Wanderung von Kationen in geschmolzenen (bzw. festen) Halogensalzen haben Klemm und verschiedene Mitarbeiter festgestellt, daß der Masseneffekt $\mu$ nach der folgenden Regel von den Ionenmassen abhängig ist ${ }^{1}$ :

$$
\mu=\frac{\Delta w}{w} / \frac{\Delta m}{m}=-0,15\left(1+\frac{1}{2,1} \frac{m^{+}}{m^{-}}\right)^{-1}
$$

wo $w$ die Wanderungsgeschwindigkeit des Ions mit der Masse $m, m^{+}$und $m^{-}$die Masse des Kations bzw. des Anions ist. Bisher sind eine Reihe von Chloriden ( $\mathrm{LiCl}, \mathrm{CuCl}, \mathrm{ZnCl}_{2}, \mathrm{AgCl}$ und $\mathrm{TlCl}$ ) und ein Bromid $\left(\mathrm{ZnBr}_{2}\right)$ in geschmolzenem Zustand und ein Jodid (AgJ) in festem Zustand untersucht worden $^{1,2}$, und alle diese Salze folgen der Regel mit der erwarteten Genauigkeit. Wir stellten uns die Aufgabe zu untersuchen, ob diese Regel auch bei anderen Salzen als Halogeniden gilt.

Hoover und Holloway haben Isotopenanreicherungen in geschmolzenem Lithiumnitrat durchgeführt $^{3}$. Sie haben aber den Masseneffekt nicht bestimmt. Wir haben nun eine Bestimmung von dieser Größe für Kaliumnitrat gemacht.

Kaliumnitrat schmilzt bei $334^{\circ}$ und hat bei unserer Arbeitstemperatur, $360^{\circ}$, eine Leitfähigkeit von $0,7 \Omega^{-1} \mathrm{~cm}^{-1}{ }^{4}$. Die Versuchsanordnung ist in Abb. 1 dargestellt. In dem Elektrolysiergefä $ß$ aus Supremaxglas sind die beiden Elektrodenräume durch ein Trennrohr (Länge $22 \mathrm{~cm}$, Innendurchmesser 4,1 $\mathrm{mm}$ ) verbunden, welches zwischen zwei Filterplatten Quarzpulver (Fraktion zwischen den Siebweiten DIN 24 und 30) enthält. Das vorher

${ }^{1}$ A. Klemm, E. Lindholm u. A.Lundén, Z. Naturforschg. $7 \mathrm{a}, 560$ [1952].

2 S. Floberg, A. Klemm u. C. Lang, Z. Naturforschg. 8a, 562 [1953]; A. Lundén u. E. Berne, Z. Naturforschg. 9 a, 684 [1954].

${ }^{3}$ J.I.Hoover u. G.E. Hollow y , NRL3897 [1951]. unter Vakuum geschmolzene Kaliumnitrat wurde in so großer Menge ins Gefäß eingegossen, daß es etwa $3 \mathrm{~mm}$ über der oberen Filterplatte des Trennrohres stand. Die Elektroden wurden dann eingeführt. Ein Platindraht diente als Anode, und als Kathode wurde ein Aluminiumrohr verwendet. Es hatte einen Durchmesser von $8 \mathrm{~mm}$ und tauchte etwa $10 \mathrm{~mm}$ in die Schmelze ein. Durch dieses Rohr wurde während des Versuches ein Gemisch von Stickstoffdioxyd und Sauerstoff dauernd

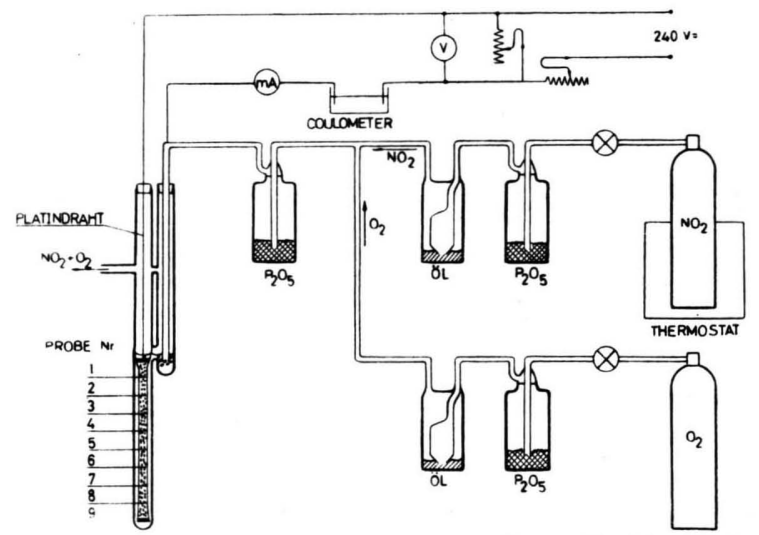

Abb. 1. Die Versuchsanordnung (schematisch). Elektrolysiergefä $\beta$, Gasversorgung und elektrische Kopplung.

durchgeblasen. Das an der Kathode abgegebene Kaliummetall wurde momentan durch Reaktion mit diesem Gasgemisch in Kaliumnitrat rückgebildet $\left(2 \mathrm{~K}+2 \mathrm{NO}_{2}+\mathrm{O}_{2}=2 \mathrm{KNO}_{3}\right)$. Die Salzmenge blieb deswegen während des Versuches konstant. Das Stickstoffdioxyd ${ }^{5}$ kam von einem Stahlbehäl-

4 Landolt - Börnstein, Physikalisch - Chemische Tabellen (5. Aufl.) Hw II, 1062.

${ }^{5}$ Wir haben dieses Gas durch freundliches Entgegenkommen von Stockholms Superfosfat AB, Ljungaverk, bekommen. Wir danken an dieser Stelle den Herren Ing. G. Morell u. Civiling. C. G, Carls son für ihre Hilfe in dieser Angelegenheit. 
ter, dessen Temperatur in einem Thermostaten konstant gehalten wurde. Nach Trocknung in einer Flasche mit Phosphorpentoxyd brodelte das Gas durch Paraffinöl, wodurch wir die Strömungsgeschwindigkeit abschätzen konnten. Es wurde mit Sauerstoff aus einem Stahlzylinder gemischt. Das Gas ging durch noch eine Flasche mit Phosphorpentoxyd, ehe es durch das Kathodenrohr strömte. Vorversuche hatten nämlich gezeigt, daß es wesentlich war das Gas sorgfältig zu trocknen, wenn man die Salzschmelze die ganze Zeit klar und durchsichtig haben wollte.

Bei den Vorversuchen wurde einige Male während längerer oder kürzerer Zeit ausschließlich Sauerstoff durch die Kathode eingeleitet. Wir bekamen dann eine weiße Ausflockung, die nur sehr langsam bei der Einführung von großen Stickstoffdioxydmengen wieder verschwand. Wenn wir nach diesen Versuchen das Salz aus dem Kathodenraum lösten, bekamen wir eine stark alkalische Lösung. Dieses bestätigte unsere Vermutung, daß die Ausflockung Kaliumoxyd war. Es ist möglich, daß man mit feuchtem Gas auch Hydroxyd bekommt.

Bei dem Hauptversuch arbeiteten wir mit einer Stromstärke von $100 \mathrm{~mA}$, was einer Stromdichte von $40 \mathrm{~mA} / \mathrm{cm}^{2}$ an der Kathode und 1,5 A/ $\mathrm{cm}^{2} \mathrm{im}$ Trennrohr entsprach.

Es wäre möglich gewesen, mit größeren Stromstärken $\mathrm{zu}$ arbeiten, aber die Vorversuche hatten gezeigt, daß man dann eine Neigung zu Gelbfärbung der Schmelze (vielleicht durch höhere Oxyde wie $\mathrm{KO}_{2}$ ) und Niveaudifferenzen zwischen den beiden Schenkeln des Gefäßes bekommt, was besondere Maßregeln verlangt, wenn man ein konstantes Niveau im Anodenraum halten will ${ }^{6}$.

Die Zellenspannung war 82 Volt. Der Versuch wurde nach sechs Tagen unterbrochen. Die durchgegangene Ladungsmenge wurde mit einem Kupfercoulometer zu 52600 Coulomb bestimmt. Sie wurde auch mit Uhr und Ampèremeter kontrolliert.

Die erkaltete Apparatur wurde in neun Stücke zerlegt (vgl. Abb. 1). Da die Salzmenge des Anodenraumes (etwa $0,3 \mathrm{~g}$ während des Versuches) viel kleiner als die des Kathodenraumes (98 g) war, erwarteten wir, daß nur im oberen Stück des Trennrohres eine meßbare Änderung in der Isotopenhäufigkeit auftreten würde. Da wir mit Kaliumnitrat von pro analysi Qualität (B. D. H. Anala R)

${ }^{6}$ Vgl. A. Klemm, Z. Naturforschg. 6a, 512 [1951].

${ }^{7}$ Wir danken British Drug Houses für die Mitteilung, daß die von uns verwendete $\mathrm{KNO}_{3}$-Verpackung $0,086 \%$ Natrium enthielt. arbeiteten, konnten wir bei der Analyse von dem Vorhandensein von anderen Kationen absehen ?

Die neun Teilstücke wurden einzeln gewogen, ehe das Salz mit Wasser ausgelöst wurde. Das nachfolgende Wägen des Glases gab das Gewicht des Salzes. Durch wiederholtes Eindampfen wurde das Nitrat in Chlorid überführt. Das Kaliumchlorid wurde gewogen. Ein Vergleich mit den durch die Differenzenbildung erhaltenen Werten zeigte so gute Übereinstimmung, daß wir von der Annahme einer Bildung von z. B. Kaliumnitrit durch Nebenreaktionen bei der Neubildung von Kaliumnitrat an der Kathode absehen konnten. (Die durch Differenzbildung erhaltenen Mengen von Kaliumnitrat sind in Tab. 1 angegeben.) Die große Salzmenge des Kathodenraumes wurde auch in Wasser gelöst. Die Lösung hatte ein $p_{\mathrm{H}}$ von 7,5 . Wir konnten darum auch von der Annahme einer Bildung von Oxyden absehen (vgl. die Vorversuche). Die Aluminiumkathode zeigte nach dem Versuch keine nennenswerten Spuren von Korrosion. Wir machten trotzdem eine Analyse, um festzustellen, ob eventuell Aluminium in dem Salz des Kathodenraumes vorhanden sei. Durch Ausfällen mit 8-Hydroxychinolin fanden wir 0,01 g Aluminium $^{8}$. Die Kathode war darum weder von der Salzschmelze noch von dem Gasgemisch angegriffen worden. Das Quarzpulver des Trennrohres wurde unter dem Mikroskop besichtigt, wobei wir keinen Angriff von der Salzschmelze beobachten konnten.

Die massenspektroskopische Analyse wurde mit einem Massenspektrometer ähnlicher Bauart wie das von Brewer ${ }^{9}$ durchgeführt. Das Instrument wird in einer später erscheinenden Veröffentlichung näher beschrieben werden. Hier werden nur einige Besonderheiten in bezug auf die Empfindlichkeit und Meßgenauigkeit kurz erörtert werden.

Das zu untersuchende Material ( $\mathrm{KCl}$ ) wurde durch Lösen in einer äquivalenten Menge verdünnter Phosphorsäure und darauf folgendes Eindampfen und Erhitzen in $\mathrm{K}_{3} \mathrm{PO}_{4}$ überführt. Das Eindampfen geschah direkt auf dem Platinband, welches als Ionenquelle diente. Die Menge Kalium, die so zur Analyse gelangte, wird auf wenige Mikrogramm geschätzt. Nach Abschluß der Analyse wurde das Glühband durch Wasserdampf und nachfolgendes Ausglühen im Vakuum wieder gereinigt.

Es kam ein neues Meßverfahren ${ }^{10}$ zur Anwendung, welches es gestattet, eine Meßgenauigkeit von $0,1 \%$ bequem zu erreichen. Es ist dies ein Kompensationsverfahren, wodurch die schwachen Ionenströme zweier Isotope direkt miteinander verglichen werden. Die Ströme gelangen aber nicht, wie bei solchen Verfahren üblich, gleichzeitig zum Meßkreis, sondern werden $a b$ wechselnd eingeschaltet. Das Umschalten der Isotopenströme wurde durch Veränderung der magnetischen

${ }^{8}$ F. P. Treadwell u. W. T. Hall, Analytical Chemistry, Vol. II, S. 151, 9 th Ed. New York 1945.

${ }^{9}$ A. K. Brewer, J. Amer. Chem. Soc. 58, 365 [1936].

${ }^{10}$ C. Reuterswärd, Ark. Fys. 8, 18 [1954] (kurzer Sitzungsbericht). 
Feldstärke bewirkt. Meßreihen desselben Tages zeigten eine Streuung der Mittelwerte aus zehn Messungen, die gewöhnlich kleiner als $0,05 \%$ war. Die Meßergebnisse von verschiedenen Tagen aber konnten zehnmal gröBere Abweichungen aufweisen. Der wahrscheinliche Fehler einer Analyse wird infolgedessen auf 0,4\% geschätzt.

Da das Spektrometergehäuse nicht durch Ausheizen von Resten des alten Analysenmaterials befreit werden kann, könnten diese, indem sie durch Wiederverdampfen zum Glühband zurückkehren, die Messungen fälschen. Um einen etwaigen Einfluß der vorausgegangenen Analysen abzuschätzen, wurden die Proben in verschiedener Folge analysiert. Das Ergebnis war, daß die vorausgegangene Probe noch zu rund 10\% mitwirkte, weswegen die Analysen der mittleren ProbenNummern entsprechend korrigiert wurden. Die extremen Proben (Nr. 1 u. 2) aber wurden wiederholt analysiert, um womöglich die Einwirkung von anderen Proben zu eliminieren. Alle Meßergebnisse (sechs je Probe) wurden in Intervallen von $1,5 \%$ erhalten, und es erscheint wenig wahrscheinlich, da $\beta$ diese Fehlerquelle die entsprechenden Zahlen der Tab. 1 mit mehr als $1 \%$ fälschen könnte. Die angeschriebenen Fehlergrenzen geben die statistisch wahrscheinlichen Fehler.

\begin{tabular}{|c|c|c|c|}
\hline Probe-Nr. & $\begin{array}{c}\text { Länge } \\
(\mathrm{mm})\end{array}$ & $\begin{array}{c}\mathrm{KNO}_{3} \\
\mathrm{mg}\end{array}$ & $\begin{array}{c}\text { Gehalt an } \\
{ }^{41} \mathrm{~K} \%\end{array}$ \\
\hline 1 & - & 316,1 & $7,64 \pm 0,03$ \\
2 & 25 & 239,6 & $7,60 \pm 0,03$ \\
3 & 14 & 155,1 & $7,15 \pm 0,03$ \\
4 & 24 & 267,8 & $6,86 \pm 0,03$ \\
5 & 36,5 & 422,5 & $6,72 \pm 0,03$ \\
6 & 36 & 413,3 & $6,71 \pm 0,03$ \\
7 & 11 & 132,4 & $6,71 \pm 0,03$ \\
8 & 34 & 450 & $6,68 \pm 0,03$ \\
9 & 28 & 589,5 & $6,69 \pm 0,03$ \\
\hline
\end{tabular}

Tab. 1. Meßergebnisse.

Bei der Berechnung des Masseneffekts setzen wir voraus, daß natürliches Kalium $6,70 \%{ }^{41} \mathrm{~K}$ enthält und daß wir in den Proben sechs bis neun die natürliche Isotopenhäufigkeit haben. Für alle Proben rechnen wir mit $0,01 \%{ }^{40} \mathrm{~K}$, da die eventuell vorhandene Änderung des ${ }^{40} \mathrm{~K}$-Gehaltes vernachlässigbar ist. Die relative Differenz der Wanderungsgeschwindigkeiten $\Delta w / w$ der Kalium-Isotope in bezug auf die Nitrationen wurde mittels der Gleichung ${ }^{11}$

$$
\frac{\Delta w}{w}=\left(\frac{{ }^{41} N}{{ }^{41} N_{0}}-\frac{{ }^{39} N}{{ }^{39} N_{0}}\right) \cdot \frac{F \cdot N_{\mathrm{K}}}{L} \text { bestimmt. }
$$

Dabei ist $N_{\mathrm{K}}$ die in Molen gemessene $\mathrm{KNO}_{3}$-Menge in den Proben eins bis fünf. ${ }^{41} N$ und ${ }^{39} N$ sind die entsprechenden Mengen der Isotope ${ }^{41} \mathrm{~K}$ und ${ }^{39} \mathrm{~K}$, während ${ }^{41} N_{0}$ und ${ }^{39} N_{0}$ der natürlichen Isotopenzusammensetzung in den fünf Proben entspricht. $L$ ist die transportierte Ladungsmenge und $F$ die

${ }^{11}$ A. Klemm, Z. Naturforschg. 1, 252 [1946].
Faradaysche Ladung. Wir bekamen 0,0724 für den Klammerausdruck und 0,0139 für $N_{\mathrm{K}}$. Damit folgt

$$
\frac{\Delta w}{w}=0,00184 \text {. }
$$

Der Masseneffekt wird dann

$$
\mu=\frac{\Delta w}{w} \cdot \frac{m}{\Delta m}=-0,036 .
$$

Ein Vergleich mit der in der Einleitung erwähnten Regel für Halogenide ist interessant. Für $m^{+}=39,1\left(\mathrm{~K}^{+}\right)$und $m^{-}=62\left(\mathrm{NO}_{3}^{-}\right)$bekommen wir $\mu_{\text {ber }}=-0,115$, d. h. einen dreimal größeren Wert als den, welchen wir experimentell gefunden haben. Es ist sehr unwahrscheinlich, daß diese Differenz in einem größeren Ausmaße von Meßfehlern herrührt. Von den Größen, die von Bedeutung für die Berechnung von $\Delta w / w$ sind, ist $L$ viel genauer als $1 \%$ und $N_{\mathrm{K}}$ wenigstens mit derselben Genauigkeit bestimmt. Wenn größere Mengen Kaliumnitrat während der sechs Tage von der Oberfläche im Anodenraum verdampft wären, bedeutete dies, daß unser experimenteller $\mu$-Wert zu klein wäre. In Probe 1 sind aber 13,8 mg Kaliumnitrat eingerechnet, die in dem über der Schmelze befindlichen Teil des Anodenschenkels sich angesammelt hatten. Es ist nicht wahrscheinlich, daß mehr als höchstens einige mg Kaliumnitrat von dem Gasstrom weiter transportiert worden sind. Dieser Effekt kann darum allerhöchst ein paar Prozent Änderung des $\mu$-Wertes bedeuten. Die größte Fehlerquelle bei der Berechnung von $\Delta w / w$ liegt ja, wie eine kleine Rechnung zeigt, in dem Klammerausdruck $\left.{ }^{41} N /{ }^{41} N_{0}-{ }^{39} N /{ }^{39} N_{0}\right]$. Wir haben aber schon gezeigt, daß die Meßfehler bei der Massenanalyse so klein sind, daß auch die Unsicherheit in dem Klammerausdruck keine größere Rolle spielt. Es ist darum deutlich, daß wenigstens der Masseneffekt des Kaliumnitrats der ,Halogenidregel“" nicht folgt. Noch nicht abgeschlossene Messungen mit Lithiumnitrat ${ }^{12}$ scheinen ein ähnliches Resultat zu ergeben, aber es ist noch zu früh, die allgemeinen Verhältnisse in Nitratschmelzen zu diskutieren. Eine weitere Behandlung muß darum anstehen, bis wir $\mu$-Werte für noch ein paar Nitrate bestimmt haben.

Wir sind dem schwedischen Atomkomitée für finanzielle Unterstützung und den Herren Professoren N. Ryde, P. Ohlin und K. Siegbahn, an deren Instituten in Göteborg und Uppsala die Arbeit durchgeführt worden ist, für ihr freundliches Interesse zu Dank verpflichtet.

12 A. Lundén, E. U. Monse u. N. Sjöberg, noch nicht abgeschlossene Untersuchung. 